\section{Cahiers de Narratologie}

Analyse et théorie narratives

$34 \mid 2018$

Les avatars du chapitre en bande dessinée

\title{
Chris Ware, une œuvre entre segmentation et agrégation
}

\section{Côme Martin}

\section{(2) OpenEdition}

\section{Journals}

\section{Electronic version}

URL: http://journals.openedition.org/narratologie/8805

DOI: 10.4000/narratologie.8805

ISSN: $1765-307 X$

Publisher

LIRCES

\section{Electronic reference}

Côme Martin, "Chris Ware, une œuvre entre segmentation et agrégation », Cahiers de Narratologie [Online], 34 | 2018, Online since 21 December 2018, connection on 01 May 2019. URL : http:// journals.openedition.org/narratologie/8805 ; DOI : 10.4000/narratologie.8805

This text was automatically generated on 1 May 2019.

Article L.111-1 du Code de la propriété intellectuelle. 


\title{
Chris Ware, une œuvre entre segmentation et agrégation
}

\author{
Côme Martin
}

\section{Introduction}

1 Il est inutile de rappeler en quoi l'œuvre de Chris Ware est majeure pour la bande dessinée anglophone, voire la bande dessinée contemporaine dans son ensemble; il peut être utile en revanche de résumer la génétique de cette œuvre, dont n'ont été publiés que deux récits complets (Jimmy Corrigan en 2000 et Building Stories en 2012'1) et deux recueils de planches semi-autonomes (Quimby the Mouse en 2003 et The Acme Novelty Library Final Report To Shareholders en 2005). Avant ces publications, les planches de Ware ont été publiées au sein de l'Acme Novelty Library, collection hybride conçue et publiée par l'auteur lui-même (pour les derniers numéros, tout du moins) qui compte aujourd'hui 20 volumes et demi, le premier étant paru en 1993 et le dernier en date en 2010. Cependant, il faut ajouter à cette bibliographie officielle d'une part au moins deux ouvrages publiés par Ware avant 1993 (Lonely Comics \& Stories en 1992, sorte de précurseur de l'Acme Novelty Library et Floyd Farland, œuvre de jeunesse publiée en 1987), d'autre part un système de prépublication dans les journaux qui remonte à 1986 et au journal The Daily Texan (dont de nombreux strips restent aujourd'hui inédits) et qui se poursuit aujourd'hui, entre autres dans le Chicago Reader et le New Yorker.

2 Il est important d'avoir à l'esprit cette hiérarchie d'une œuvre en trois niveaux de publication (des journaux à l'Acme Novelty Library aux recueils, avec un décalage de plusieurs années entre chaque étape) car elle informe le rapport de Chris Ware au chapitrage de ses récits, c'est-à-dire à leur découpage en segments plus ou moins indépendants puis à leur agrégation en un tout cohérent. Cette oscillation entre segmentation et agrégation est d'autant moins anodine qu'on sait que Ware conçoit ses livres comme des tout esthétiques, où l'apparence et l'aspect matériel sont des critères primordiaux. L'étude des volumes de l'Acme Novelty Library semble sur ce point essentielle tant il est possible de les lire à travers la figure du chapitre : malgré leur contenu et leur 
format disparates, ils sont conçus comme faisant partie d'une série et constituent dès lors des objets à la fois transitoires et autonomes. Il sera donc question en premier lieu de l'analyse de ces volumes, avant d'étudier le chapitrage des divers volumes de Ware (Jimmy Corrigan, Quimby the Mouse et le Final Report) : comment ces œuvres ont-elles été agrégées à partir de leur prépublication et quel est l'effet à la lecture de leur regroupement au sein d'œuvres denses et néanmoins chapitrées ? Enfin, une troisième partie s'intéressera à la tension entre hétérogénéité et cohésion propre à Building Stories, l'œuvre la plus explicitement chapitrée de Ware et pourtant également celle qui bouleverse le plus les modalités de lecture auxquelles nous sommes habitués. Ces trois parties sont à envisager comme une sorte de tour d'horizon de la façon dont l'œuvre de Ware propose de façon quasi-exhaustive l'éventail des possibilités qu'offre le chapitrage en bande dessinée.

\section{L'Acme Novelty Library comme lieu transitoire et comme lieu de rassemblement}

$3 \mathrm{Au}$ vu de la genèse résumée ci-dessus, l'Acme Novelty Library apparaît comme le point de jonction entre la prépublication dans les journaux de l'œuvre de Ware - qui se faisait une planche à la fois dans le cas de Quimby the Mouse et deux planches à la fois dans le cas, par exemple, de Jimmy Corrigan ${ }^{2}$ - et la publication du recueil d'un récit à proprement parler. Ce processus de publication n'est pas anodin dans le cadre d'une discussion sur le chapitrage car il montre non seulement l'œuvre en train de se faire mais également l'œuvre en train de se structurer.

4 De nombreuses différences, parfois minimes, existent au sein d'une même planche depuis sa publication dans un journal jusqu'à sa forme définitive dans un recueil : le titre d'un récit peut légèrement changer, le texte d'une lettre (dans Jimmy Corrigan) être modifié, des détails moins anodins qu'ils n'y paraissent car ils marquent la rupture entre une œuvre largement improvisée au fil des volumes et un récit structuré et réfléchi. Même l'aspect matériel des volumes en témoigne: jusqu'au numéro 8, les numéros de l'Acme Novelty Library sont de format et de contenu très divers, puis Chris Ware décide d'y sérialiser le récit de Jimmy Corrigan ; jusqu'au numéro 12, les numéros sont d'une fabrique relativement modeste, couvertures souples auxquelles les pages sont agrafées, avant qu'elles soient thermocollées dans les numéros 13 et 14 (qui contiennent sensiblement plus de pages) et que la couverture des volumes devienne rigide et la reliure cousue à partir du numéro 16.

5 C'est que, de simple support de publication pour les récits hétérogènes de Ware, l'Acme Novelty Library va peu à peu tordre son appellation ironique pour se constituer en véritable bibliothèque de l'auteur, selon les termes d'Ugo Dionne. Dans La Voie aux chapitres, Dionne rappelle en effet :

La Bibliothèque est une variation sur le thème de la collection. [...] Le disparate des ouvrages compilés n'empêche pas - et tendrait même plutôt à encourager - les efforts d'harmonisation de l'ensemble : chaque roman est écrit (ou, le plus souvent, réécrit) pour la série dans laquelle il trouve place; il est raboté jusqu'à ce qu'il s'insère sans accroc dans la totalité organique de la Bibliothèque, comme un volume sur son rayon. (Dionne $2008: 64$ )

6 Cette harmonisation d'un ensemble disparate est particulièrement visible dans la façon dont les numéros successifs de l'Acme Novelty Library sont réorganisés par Ware en différents volumes : ainsi le volume 1 correspond aux travaux les plus anciens de l'auteur 
(autour de la figure du "Potato Guy»), le volume 2 aux planches de "Quimby the Mouse ", le volume 3 à celles de "Sparky the Cat ", le volume 4 à une histoire à part de Jimmy Corrigan, les volumes 5 et 6 aux chapitres de Jimmy Corrigan et le volume 7 aux deux «Joke Books» qui constitueront la colonne vertébrale de l'Acme Novelty Library Final Report.

Ce n'est donc pas un hasard si Jacques Samson qualifie l'Acme Novelty Library de « fonds catalogué » (2010: 109) ; il s'agit bien avec ces volumes d'amener une cohésion à des travaux disparates qui n'étaient pas nécessairement prévus originellement comme chapitres de ce qu'on pourrait qualifier de méta-œuvre globale. Il faut en effet garder en tête la mythologie que Ware construit autour de l'Acme Novelty Library, usine à bandes dessinées dont la visite est proposée au lecteur à la fois dans le numéro 3 et dans le Final Report. Réagencer l'ordre des volumes pour qu'il soit chronologique, c'est les insérer dans une Bibliothèque au sens que lui donne Ugo Dionne, qui écrit par ailleurs que «l'œuvre singulière ne prend tout son sens que lorsqu'elle est mise en relation avec la totalité dont elle fait partie » $(2008: 138)$. Je ne suis pas aussi catégorique, mais force est de constater que Ware organise bien les volumes de l'Acme Novelty Library comme une cosmogonie, ainsi que le révèle le diagramme (fig. 1) présent dans le volume 20 de la collection.
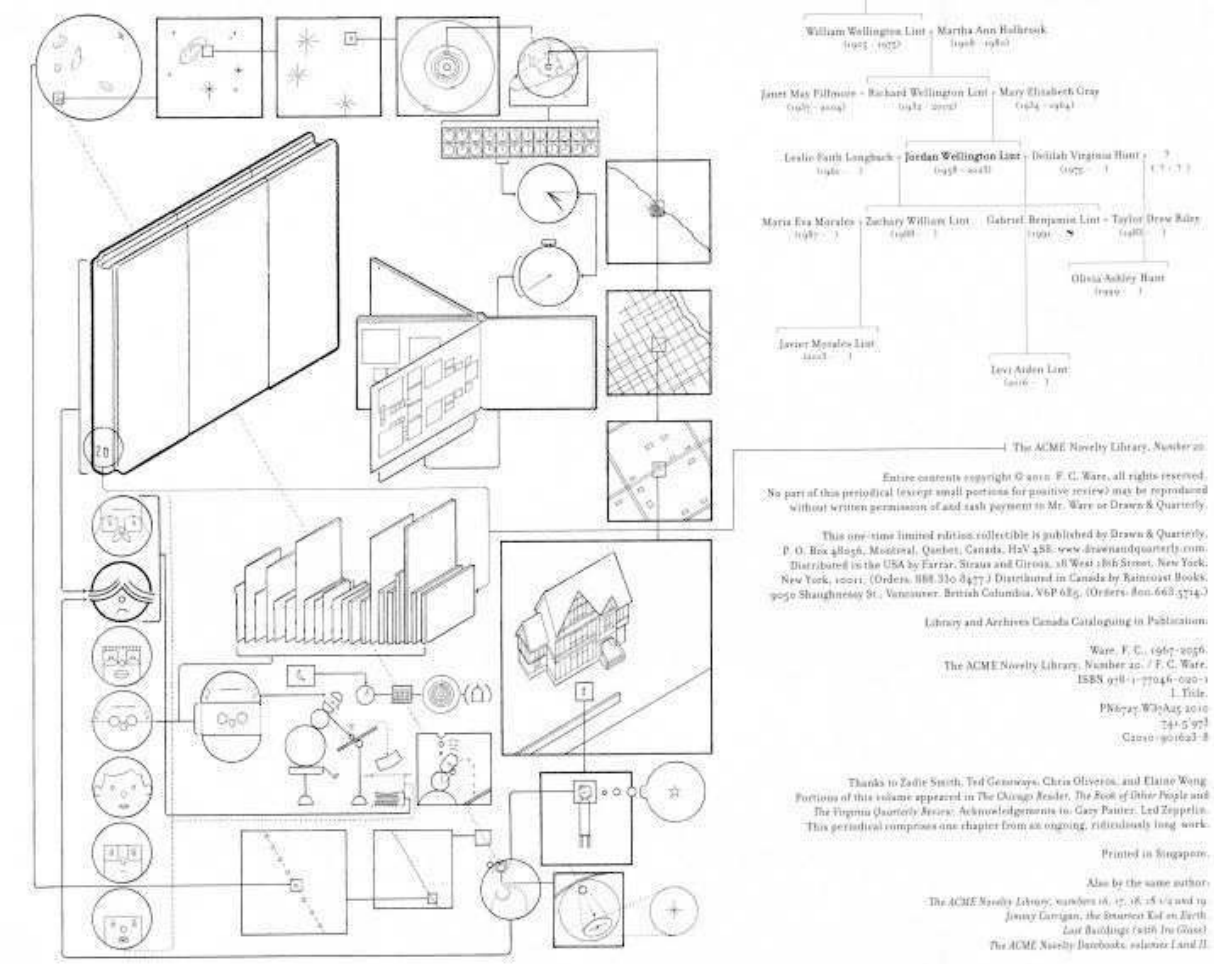

Chris Ware, Acme Novelty Library $n^{\circ} 20$

Reproduit avec l'accord de l'auteur, copyright @ 2018 Chris Ware

Pour résumer, le chapitrage au sein de l'Acme Novelty Library semble se faire sous le prisme de deux principes : la révision et la périodicité. La révision car, comme l'ont noté Eugene Kannenberg d'une part (2002: 174-175) et Gary Groth d'autre part (1997: 130), lorsque les planches publiées éparses dans les journaux sont collectées en volumes, elles peuvent être partiellement redessinées et leur ordre modifié, à l'inverse de la grande majorité des prépublications de récits dessinés. Ware n'hésite pas à rendre plus cohérents des récits qui, de son propre aveu, étaient souvent improvisés et manquaient donc parfois 
de logique, ou de rythme (Groth 1997 : 135). Ainsi le récit n'est-il jamais tout à fait figé et, comme l'écrit Daniel Raeburn, il continue de changer même une fois qu'il est terminé (1999: 2). D’autre part, la périodicité des volumes a également un rôle crucial dans la segmentation de l'œuvre, comme l'écrit Jacques Samson :

Le véritable dénominateur commun de l'ANL n'est pas à rechercher dans les publications elles-mêmes mais dans le principe éditorial qui les gouverne toutes, sans exception, à savoir la périodicité ou le mode de publication feuilletonesque. Bien qu'adoptant à l'occasion (et de plus en plus souvent) une forme se rapprochant parfois du graphic novel, et assimilable au livre plutôt qu'au périodique, ces publications ont à peu près toujours mêlé des bandes dessinées avec divers matériaux rédactionnels d'abord parus dans l'un ou l'autre des journaux auxquels Ware collabore depuis ses débuts. (Samson $2010: 111-112$ )

En effet, il est primordial de mentionner l'appareil pseudo-journalistique qui accompagne la majorité des volumes de l'Acme Novelty Library, transformant chaque numéro en entité indépendante, au lieu de considérer la livraison comme un chapitre d'un récit plus long. Ce sont en grande parties ces fausses publicités, courrier des lecteurs, éditoriaux et autres éléments péritextuels qui créent la tension évoquée ci-dessus entre segmentation et agrégation, d'autant plus que ces textes, s'ils individualisent chaque numéro de l'Acme Novelty Library, permettent également de rendre cohérent des ensembles disparates, ainsi en va-t-il des planches qui constituent le volume de Quimby the Mouse.

\section{Segmentation, chapitres et regroupements dans les volumes de Ware}

10 C'est bien par le texte que s'effectue la suture du propos dans Quimby the Mouse, recueil publié en 2003 qui reprend les planches de l'Acme Novelty Library numéro 2 et 4, ainsi que quelques planches du Lonely Comics and Stories. Regrouper des planches autour des personnages de Quimby the Mouse et Sparky the Cat, respectivement, dans ces deux livraisons de l'Acme Novelty Library était une façon de proposer au lecteur deux volumes thématiques; le recueil de Quimby the Mouse va bien plus loin en transformant des planches «absurdes, surréalistes, répétitives et disjointes ", pour citer Benjamin Widiss $\left.(159, \text { m.t. })^{3}\right)$ en un témoignage autobiographique, grâce à l'essai de plusieurs milliers de mots qui introduit l'ouvrage. Il devient alors possible de lire ce qui ne semblait qu'un hommage à Krazy Kat sous l'angle autobiographique et d'y discerner à la fois une fictionnalisation des rapports amoureux de Ware et de sa relation avec sa grand-mère. Cette lecture n'est rendue possible que parce que les planches sont devenues volumes, comme l'écrit Widiss :

Par-dessus et contre la nature singulière et réitérative des strips individuels, il est possible d'interpréter ces derniers comme une narration minimale dans laquelle la deuxième tête de Quimby flotte à l'article de la mort pendant quelques temps puis disparaît, laissant derrière elle un Quimby en solo, qui prend ensuite Sparky sous son aile, peut-être dans un effort de compensation, avant d'essayer de manière répétée de se débarrasser d'elle également. Ware confirme cette séquence englobante dans l'introduction où il indique que la première partie des planches [celles qui correspondent au volume 2 de l'ANL] ont été dessinées au Texas à la fin de 1990, alors que sa grand-mère bien-aimée était mourante, et la deuxième partie pendant les six mois suivants, alors qu'il envisageait puis mettait à exécution un 
déménagement vers Chicago, «laissant derrière [lui...] une relation qu'[il] avait complètement ruinée à cause d'un comportement égoïste et égocentrique.» (Widiss 2010 : 162-164, m.t.)

11 La transformation d'un simple recueil de planches expérimentales en volume autobiographique se fait donc à l'aide d'une confession écrite dont on ne trouve quasiment aucun équivalent dans tout le reste de l'œuvre de Ware, mis à part peut-être dans le «Corrigenda » qui clôt Jimmy Corrigan et qui établit un parallèle entre la genèse de l'œuvre et la rencontre de l'auteur avec son père. De façon plus cruciale, c'est ce texte qui divise les planches de Quimby the Mouse en deux époques, deux chapitres d'un récit luimême constitué de segments plus courts. Une stratégie similaire est observable dans l' Acme Novelty Library Final Report To Shareholders, qui constitue une collection de planches plus diverses encore puisqu'Allen Rubinstein y a recensé des extraits des numéros $2,3,5$, $6,7,8,9,10,11,12$ et 15 avec beaucoup de planches omises et certaines dans un ordre ou une colorisation différente (2-4), ce qui témoigne une fois de plus du soin qu'a pris Ware à remonter ces planches en volume (les planches des volumes 7 et 15 ayant déjà fait l'objet d'une drastique sélection par rapport à leur prépublication, comme l'indiquent certaines pages des carnets de croquis de Ware, les Acme Novelty Datebooks, sur lesquelles il réfléchit à la cohérence à donner aux deux volumes).

Il faudra un lecteur attentif pour remarquer l'organisation du livre en un cycle allant de la création du monde et la jeunesse des personnages à leur vieillesse et à des temps postapocalyptiques. L'ensemble s'articule autour d'un récit cyclique, le seul qui ne soit pas indiqué dans la table des matières de l'ouvrage: il représente le personnage de Dieu / Superman, récurrent dans l'œuvre de Ware, dans ses tentatives de création du monde et de quête du bonheur. On peut en effet déceler, sur la deuxième de couverture (qui présente une série de fausses publicités) une case en bas à droite dans laquelle Dieu flotte dans l'espace, un début de récit qui se déploie sur sept cases au sein d'autres publicités dans la double page suivante, puis à nouveau un strip de six cases représentant Dieu en train de placer des étoiles dans l'espace vide (de la case, et du monde). Ces prolégomènes ne sont pas anodins car non seulement ils se prolongeront à plusieurs reprises dans l'ouvrage, mais de plus cette création d'étoiles laisse place, aux pages 4 et 5 , à une constellation phosphorescente, puis à la création du monde et à une série de strips sur l'histoire de la création picturale. Par son apparition progressive et discrète qu'on pourrait croire fortuite, le personnage de Dieu sert en réalité à l'auteur de passerelle entre ce qui n'est plus tout à fait le paratexte (car parasité par un récit sériel et épisodique) et le corps de l'ouvrage, lui-même composé d'histoires qui pourraient paraître sans lien les unes avec les autres mais qu'on peut rattacher à ce même récitcadre de création puis de disparition du monde. Ce lien entre les planches de l'ouvrage, bien que discret, est primordial car il témoigne ici de la volonté de l'auteur de faire de ce Final Report la somme totale et englobante de sa production au sein de l'Acme Novelty Library, une sorte de ménage de printemps avant le début de la sérialisation de Rusty Brown à partir de l'Acme Novelty Library numéro 16, comme en témoigne l'adjectif « Final » du titre du volume.

13 Le regroupement de ces planches selon des thèmes communs correspond par ailleurs à l'un des trois effets que Charles Hatfield attribue à la publication en volume de bandes dessinées précédemment sérialisées : la répétition d'un thème ou d'une même structure de discours va amener une forme et une continuité au récit global (2005: 154-159). En effet, avec ses fausses publicités, ses pages à découper et autres faux appareils 
paratextuels, le Final Report refuse d'effacer ses origines sérielles et chapitrées, comme l'écrit Matthieu Letourneux à propos de la publication en recueils de Fantômas :

Lors même que la série quitte l'espace de la presse, [l'auteur] [fait] des productions journalistiques des intertextes majeurs [...]. Tout se passe comme si, en abandonnant le support du journal et la logique qui lui était associée, la fiction devait compenser cette perte référentielle en surinvestissant la dimension médiatique de [son] imaginaire. (Letourneux 2013 : \$16)

14 Les planches de Ware, lorsqu'elles sont collectées en volumes, conservent les «trace[s] de la conception de l'œuvre pour la lecture discontinue ", pour paraphraser Jean-Christophe Menu (2010 : 304). Menu écrit dans sa thèse que dans de tels volumes, « la présence de ces éléments signifiants, même si les planches étaient conçues pour paraître d'emblée en livre, venaient constituer un simulacre de feuilleton appelant la micro-discontinuité [...] et contraignant le lecteur à une apnée dans sa lecture, brisant la linéarité, amenant des perturbations » (2010: 305). En d'autres termes, le caractère fragmenté et pourtant cohérent du Final Report amène à des pauses nécessaires dans la lecture de la même façon qu'un chapitrage dans une œuvre longue, comme l'écrit Jacques Dürrenmatt dans Bande dessinée et littérature :

On sait qu'une des raisons essentielles du chapitrage, abondamment commentée à l'époque classique, est la nécessité de ménager des pauses indispensables au repos lors de la lecture envisagée comme un voyage au long cours. Sur une "distance » brève, une division en chapitres risque donc de découper inutilement ce qu'une lecture sans pause longue saisit aisément dans sa totalité organisée, sauf en cas de récit particulièrement dense et complexe. (Dürrenmatt $2013: 105$ )

Il n'y a jamais de blanc interchapitral dans les œuvres de Ware; les respirations y sont pourtant présentes, ramassées, brèves mais indispensables à la lecture. Ware était déjà conscient du besoin de pauses dans la lecture dès Floyd Farland, son œuvre de jeunesse publiée en 1987, qui contient la postface suivante ${ }^{4}$ :

L'espace entre chaque page du livre que vous tenez à présent représente une semaine de « temps écoulé » entre les épisodes tels qu'ils sont apparus à l'origine. À cause de cette distorsion transpositionnelle (du strip vers le livre), je voulais originellement suggérer au nouveau lecteur de n'ingérer qu'une page par semaine de cette œuvre, afin de simuler efficacement l'« expérience journalistique »; bien sûr, personne ne le ferait, probablement, et même si quelqu'un essayait, il ou elle cesserait probablement rapidement de s'y intéresser. Neuf mois pour lire une bande dessinée ? (1987 : n.p. ${ }^{5}$, m.t.)

16 Floyd Farland, écrit Ware, a donc été en partie redessiné pour gommer les traces d'une sérialité originelle, ce qui n'est pas le cas du recueil de Jimmy Corrigan qui, au contraire, arbore ces mêmes traces dès les premières pages du récit, via la récurrence, presque obsessionnelle, de son titre. Celui-ci apparaît six fois en deux pages dès le début du récit (correspondant aux premières pages de l'ANL 5 dont le sous-titre est d'ailleurs «Le premier d'une série de chapitres sophistiqués format poche»). Ce titre revient deux fois dans le chapitre correspondant à l'ANL 5, deux fois dans celui qui correspond à l'ANL 6, une fois au milieu du chapitre de l'ANL 8, une fois dans l'ANL 9, aucune fois dans l'ANL 11 (on en conclut que les parties du récit concernant le grand-père de Jimmy ne sont pas titrées), une fois dans l'ANL 12, aucune fois dans l'ANL 13 et une fois dans l'ANL 14. Peuvent éventuellement s'ajouter à ce recensement les couvertures de l'ANL qui mentionnent explicitement le titre du récit : les numéros 5, 6, 9, 11 et 14 .

De cet inventaire un peu rébarbatif, il ressort que chapitres et récurrence du titre ne vont pas de pair dans Jimmy Corrigan, le récit étant divisé en sept volumes et le titre 
apparaissant quatorze fois, de façon de moins en moins récurrente. Samuel Bréan interprète cette récurrence « immodérée en taille » comme un arrêt dans la narration qui sert de "rappel de l'intitulé ironique du livre tout en étant conforme à ses thèmes " (2001 : 51, m.t.), mais il semble que, tout comme l'usage répété de cartouches de type « Et donc » dans le récit, la présence du titre n'est pas uniquement ironique. Le titre sert en effet de scansion, de point de repos, pour paraphraser Jacques Dürrenmatt (2013 : 105) ; il sert également de rappel, non pas du caractère ironique mais plutôt de l'aspect improvisé de ce récit, ce qui explique sa disparition progressive à mesure que la direction vers laquelle se dirige l'histoire se fait plus affirmée (en témoigne également la longueur des deux dernières livraisons de l'ANL). S'embarquer dans la lecture de Jimmy Corrigan, ce serait alors voir disparaître progressivement ces repères, comme les jalons familiers s'effacent en même temps que les côtes lors d'un voyage au long cours (s'il y a une ironie, c'est bien que ces jalons s'effacent à mesure que la fin du récit se rapproche). Le récit, bien que long et parsemé de pauses nettes dans la narration (on y retrouve plusieurs pages muettes, souvent en transition d'une époque à l'autre du récit), ne se laisse pas enfermer dans un chapitrage catégorique et continue d'être tendu entre ses différents points d'origine et ses fins multiples (l'édition en couverture souple ajoutant un second épilogue au récit après le "Corrigenda »). C'est, sous une autre forme, cette même tension d'un chapitre, d'un moment, d'un segment à un autre qu'explore la forme non conventionnelle de Building Stories.

\section{Le chapitrage dans Building Stories}

Inutile de présenter ici le principe structurel qui régit Building Stories tant il a été commenté6. Néanmoins, ce principe pose au moins deux questions: quel chapitrage l'œuvre adopte-t-elle en regard d'une segmentation plus conventionnelle à l'instar de celles que présentent la plupart des romans graphiques contemporains? Quel effet ce chapitrage a-t-il sur le lecteur, la lectrice et la lecture?

Il n'est pas certain que Building Stories soit une franche réaction au roman graphique ou au développement de la bande dessinée numérique: on trouve en effet dans le premier Datebook, page 50 (une page datée de 1990) un projet qui ressemble à l'ouvrage publié en 2012 et Ware indique dans un entretien avoir eu l'idée d'un « récit en boîte » dès 1987, mais sa réalisation lui fut refusée par son éditeur de l'époque (Mautner 2012 : n.p.). Building Stories, comme la plupart des projets de Ware, émane donc d'une longue gestation créative; mais à l'inverse de ses autres publications, il porte les traces de sa gestation dans sa forme publiée, comme l'écrit Benoît Crucifix :

Les objets divers et hétérogènes assemblés dans la boîte sont en lien direct avec la dépendance continue de Ware envers la sérialisation : le roman graphique [sic] est d'abord apparu, au fil d'une décennie, dans des myriades de différents lieux, ses morceaux dispersés dans divers journaux, magazines, livres, anthologies, et même sous la forme d'une application numérique. L'hétérogénéité matérielle et la structure narrative fragmentée de Building Stories renvoie à cette généalogie de sérialisation dispersée : les différents formats font écho aux formes matérielles disparates de sa sérialisation, alors que les groupes narratifs fragmentés sont en lien étroit avec ce mode de publication épisodique diluée dans le temps. (Crucifix $2017: 13$, m.t.)

20 Les quatorze fragments que contient la boîte de Building Stories ne renient pas leur histoire, au point, pour au moins l'un d'entre eux, de reproduire quasiment à l'identique 
le volume 18 de l'Acme Novelty Library ${ }^{7}$. Contrairement à la transformation des volumes de l'ANL dans les publications de Quimby the Mouse ou même de Jimmy Corrigan en volume, Ware exhibe ici la genèse de la publication de Building Stories et résout en un coup de maître la question épineuse de l'édition en un seul volume de formats si disparates : il suffisait que le volume soit une boîte au lieu d'un livre. Pour citer à nouveau Benoît Crucifix : « Le format de la bande dessinée en boîte, au contraire du livre, permet à Ware de repositionner le numéro "Building Stories" de l'Acme Novelty Library en tant que fragment distinct qui fait partie d'une plus grande mais aussi plus lâche collection d'objets, non séquencée par l'ordre des pages reliées » (2017: 14, m.t.). Le chapitrage de Building Stories est donc certes non-conventionnel par rapport à la plupart des bandes dessinées parce qu'il permet à son lecteur de saisir le récit dans l'ordre de son choix, mais sa division en fragments ne diffère de celle, par exemple, de Jimmy Corrigan, que sur un seul point : ici, la segmentation interchapitrale se mue en espace matériel et les coupes d'un chapitre à l'autre sont franches et physiques. Cela permet une disparité de formats, mais aussi de temps de lecture, puisque certains chapitres sont extrêmement courts et ne demandent que quelques minutes pour être parcourus en intégralité, là où il faudra des heures pour en traverser d'autres.

Davantage que la matérialité de son chapitrage, c'est l'effet qu'a Building Stories sur nos habitudes de lecture qui est véritablement remarquable, et qui a d'ailleurs déjà été remarqués. C'est à nouveau sur l'espace entre les chapitres qu'il convient de s'attarder puisque, comme l'écrit Torsa Ghosal :

Il y a trois niveaux principaux d'intervalles [dans Building Stories] : les intervalles ou gouttières qui séparent les différents cadres du récit graphique, les intervalles temporels dans le récit d'un fragment à un autre, et la séparation physique entre les différents documents. (Ghosal $2015:$ 82, m.t.)

La fragmentation de Building Stories fait encore davantage sens dès lors qu'elle répond à la fragmentation inhérente à la narration graphique en général, et à la narration graphique chapitrée en particulier. Les chapitres de Building Stories ne sont pas tout à fait des « unités indépendantes» comme les appelle Anne Chassagnol (2015: \$2) : ce sont plus précisément des unités interdépendantes et ce sont ces articulations d'un chapitre à un autre (dont il reviendra à un meilleur mathématicien que l'auteur de cet article de calculer le nombre de permutations possibles) qui créent la tension, une fois encore, entre fragmentation et agrégation. Avec Building Stories, le lecteur se voit offrir, en quelque sorte, les quatorze livraisons d'une même revue en une seule fois, l'assurant d'une cohérence et d'une globalité narrative qui reste absente ou fragile dans le cas des numéros de l'Acme Novelty Library.

Il faut pour finir mentionner les tensions à l'œuvre dans Building Stories quant à son temps de lecture. La question se posait déjà pour la collection de planches éparses dans le cas, notamment, du recueil Quimby the Mouse, mais comme l'écrit Benoît Crucifix :

Building Stories, à proprement parler, ne propose pas une simulation de la lecture périodique de "l'expérience journalistique ", ni ne fournit au lecteur un «flux narratif ininterrompu et une expérience unique en bande dessinée ${ }^{9}$ ». Dans la "distorsion transpositionnelle» de ses livraisons sérielles au sein d'un roman graphique, Building Stories garde dans sa collection quelque chose de l'intervalle sériel, en produisant un format de bande dessinée en boîte qui rappelle la longue pratique de lecture et de collection de la culture de la bande dessinée, tout en se présentant comme une archive de sa propre sérialisation. (2017 : 9-10, m.t.) 
ing Stories parvient donc à jouer un double jeu en gagnant sur les deux tableaux : il incarne à la fois la pratique du chapitrage et de la segmentation poussée à son extrême et l'agrégation de fragments impossibles à réunir autrement. C'est à la fois un renvoi au statut éphémère de la bande dessinée sérielle (par ses formats en journal, en dépliant) et un objet de collectionneur qui ne peut se lire qu'en dégageant le temps et l'espace nécessaire pour le faire, c'est-à-dire en adoptant une nouvelle forme de temporalité longue de la lecture.

\section{Conclusion}

Le chapitrage, dans l'œuvre de Ware, est une affaire complexe, ne serait-ce que parce que son emploi est étroitement lié à l'histoire de la publication de chaque récit. Depuis les premiers numéros de l'Acme Novelty Library jusqu'à Building Stories, pour ne rien dire des prépublications dans les journaux, l'œuvre de Ware affiche paradoxalement un goût pour la fragmentation narrative, notamment à travers un découpage plus ou moins précis en chapitres, tout en se concevant comme un tout esthétique et formel. La resegmentation des récits lorsqu'ils sont publiés en volume permet à l'auteur d'effectuer un tri et de laisser de côté les travaux qu'il ne souhaite pas intégrer dans sa mythologie personnelle, par exemple Floyd Farland, soulignant par là-même que chapitrer un ou des récits c'est également décider où ils commencent et où ils s'arrêtent. Voilà en somme le projet de Ware dans l'élaboration de sa Bibliothèque personnelle: rester à la frontière entre segmentation et agrégation, et faire confiance au lecteur pour joindre les deux bouts.

\section{BIBLIOGRAPHY}

Bréan, Samuel (2001), Jimmy Corrigan, the Smartest Kid on Earth: a Hall of Mirrors, Mémoire de maitrise, Bordeaux, Université Michel de Montaigne.

Chassagnol, Anne (2015), «L'immeuble mode d'emploi : Building Stories ou l'art de dégrafer le roman graphique américain », Revue de recherche en civilisation américaine, en ligne, consulté le 27 février 2018, URL : http://journals.openedition.org/rrca/696.

Crucifix, Benoît (2017), « From Loose to Boxed Fragments (and Back Again). Seriality, the Graphic Novel, and Chris Ware's Building Stories ", Journal of Graphic Novels and Comics, en ligne, consulté le 27 février 2018, URL : https://www.tandfonline.com/doi/full/10.1080/21504857.2017.1303619.

Dionne, Ugo (2008), La Voie aux chapitres, Paris, Seuil.

Dürrenmatt, Jacques (2013), Bande dessinée et littérature, Paris, Classiques Garnier.

Ferraz Gomes, Hugo (2014), Of Structural Denial, Lisbonne, Faculdade de Ciências Sociais e Humanas, en ligne, consulté le 27 février 2018, URL : https://run.unl.pt/ bitstream/10362/14686/1/Of\%20Structural\%20Denial\%20-\%20A\%20Narratological\%20Study\% 20of\%20the\%20Structural\%20Disintegration\%20of\%20the\%20Novel\%20Form.pdf. 
Ghosal, Torsa (2015), « Books With Bodies », StoryWorlds : A Journal of Narrative Studies, $\mathrm{n}^{\circ} 7(1), \mathrm{p}$. 75-99.

Groth, Gary (1997), « Understanding Chris Ware’s Comics », The Comics Journal, n²00, p. 118-178.

Hatfield, Charles (2005), Alternative Comics, Jackson, University Press of Mississippi.

Kannenberg, Eugene (2002), « Form, Function, Fiction : Text and Image in the Comics Narratives of Winsor McCay, Art Spiegelman, and Chris Ware ", Thèse de doctorat, Storrs, University of Connecticut.

Letourneux, Matthieu (2013), « Des feuilletons aux collections populaires », Belphégor, n¹1(1), en ligne, consulté le 27 février 2018, URL : http://journals.openedition.org/belphegor/286.

Mautner, Chris (2012), " "I Hoped that the Book Would Just Be Fun" : A Brief Interview with Chris Ware ", The Comics Journal, 10 octobre 2012, en ligne, consulté le 27 février 2018, URL : http:// www.tcj.com/i-hoped-that-the-book-would-just-be-fun-a-brief-interview-with-chris-ware/. Menu, Jean-Christophe (2010) La bande dessinée et son double, Paris, L'Association.

Samson, Jacques (2010), « Chris Ware - Une célébration de la bande dessinée », in Chris Ware, J. Samson \& B. Peeters, Bruxelles, Les Impressions Nouvelles, p. 101-144.

Raeburn, Daniel (1999), The Imp, n³.

Rubinstein, Allen (n. d.), « Acme Novelty Library Analysis ». Acme Novelty Library Archive, en ligne, consulté le 27 février 2018, URL : http://acmenoveltyarchive.org/media/pdf/

Acme_Hardcover_Analysis.pdf.

Ware, Chris (1987), Floyd Farland, Forestville, Eclipse Books.

Widiss, Benjamin (2010), «Autobiography with Two Heads: Quimby the Mouse », in The Comics of Chris Ware - Drawing is a Way of Thinking, D. M. Ball et M. B. Kuhlman (dir.), Jackson, University Press of Mississippi, p. 159-173.

\section{NOTES}

1. Un premier volume de Rusty Brown devrait paraître en 2018 ; on peut aussi raisonnablement considérer le volume 20 de l'Acme Novelty Library, intitulé Lint, comme un récit complet.

2. Pour être exact, une seule planche dans un journal devient deux planches à l'italienne dans l' Acme Novelty Library.

3. Cette indication signale que je suis l'auteur de la traduction.

4. Je remercie Benoît Crucifix de me l'avoir fait découvrir.

5. Cette indication signifie que l'œuvre n'est pas paginée.

6. Voir par exemple Crucifix (2017).

7. À l'exception du texte sur sa couverture, de ses dimensions légèrement plus grandes et de l'ajout de deux pages à l'intérieur du chapitre.

8. Voir par exemple Ferraz Gomes (2014).

9. Crucifix cite ici la postface de Floyd Farland. 


\section{ABSTRACTS}

Chris Ware's work displays an affinity for fragmentation, both in its narration and its chaptering, but remains conceived as a formal and aesthetic whole. It is particularly visible in the volumes of the Acme Novelty Library, which can nevertheless be considered as parts or even chapters of a series designed coherently. It also seems necessary to question the function of chaptering in the denser works of the author, distanced from serialized publications where pauses can be inserted, as is also the case in the radical Building Stories. In this article, I aim to explore the tension between fragmentation and aggregation in Chris Ware's work, since it appears to offer, in an almost exhaustive way, the range of possibilities allowed by chaptering in comic books.

L'œuvre de Chris Ware affiche un goût pour la fragmentation narrative et chapitrale tout en se concevant comme un tout esthétique et formel, notamment au sein des volumes de l'Acme Novelty Library. Il est néanmoins possible de considérer chacun d'entre eux selon sa place dans une série qui réfléchit sa cohérence et peut sans doute se lire à travers la figure du chapitre. D’autre part, il me semble nécessaire de m'interroger ce que le chapitrage fait à la lecture des récits denses de l'auteur par rapport à une publication par livraisons, qui ménage des espaces de respiration au cours de la lecture, tout comme cela peut être le cas dans le radical Building Stories. C'est la tension entre fragmentation et agrégation dans l'œuvre de Chris Ware que j'entends explorer ici, tant elle me paraît proposer, de façon quasi-exhaustive, l'éventail des possibilités qu'offre le chapitrage en bande dessinée.

INDEX

Keywords: Acme Novelty Library, aggregation, chaptering, Chris Ware, fragmentation, Jimmy Corrigan

Mots-clés: Acme Novelty Library, agrégation, chapitrage, Chris Ware, fragmentation, Jimmy Corrigan

\section{AUTHOR}

\section{CÔME MARTIN}

Côme Martin est docteur en littérature contemporaine américaine ; il travaille sur les relations entre texte et image et sur les formes du livre, aussi bien en bande dessinée qu'au sein du roman. Il est membre associé du laboratoire GRENA (Groupe de Recherche sur le Neuvième Art), à Paris IV - Sorbonne et du groupe de recherche TIES (Texte, Image et Son) à Paris Est - Créteil. Courriel : come.martin@gmail.com 